\title{
An Addition to Art Galleries with Interior Walls*
}

\author{
P. Hliněný \\ The Fields Institute, University of Toronto, \\ 222 College Street, Toronto, Ontario M5T 3J1, Canada \\ hlineny@member.ams.org
}

\begin{abstract}
Consider an art gallery formed by a polygon on $n$ vertices with $m$ pairs of vertices joined by interior diagonals, the interior walls. Each interior wall has an arbitrarily placed, arbitrarily small doorway. It is shown in [5] that the minimum number of guards that suffice to guard all art galleries with $n$ vertices and $m$ interior walls is $\min \{\lfloor(2 n-$ $3) / 3\rfloor,\lfloor(2 m+n) / 3\rfloor,\lfloor(2 n+m-2) / 4\rfloor\}$. The proofs for the first two bounds lead to lineartime guard placement algorithms, while this is not known for the third case. We present an alternative short proof for the third upper bound $L(2 n+m-2) / 4\rfloor$ that also leads to a linear-time guard placement algorithm.
\end{abstract}

\section{Introduction}

The original art gallery problem, posted by Klee and solved by Chvátal [2], is to find the smallest number of guards necessary to cover any simple (not necessarily convex) polygon-the art gallery, on $n$ vertices. Here a covering by $g$ guards means that one can find $g$ points in the interior of the polygon such that every point in the interior is joined with some guard by a straight-line segment not intersecting the polygon. The example in Fig. 1 can be generalized to an arbitrary number of vertices divisible by 3 , and so $[n / 3\rfloor$ guards are sometimes necessary. Chvátal showed that $\lfloor n / 3\rfloor$ guards always suffice. For more information on the history of this problem and related problems, see [6].

We now give Fisk's [3] elegant proof of Chvátal's result, that is an inspiration for our proof: First triangulate the polygon, which results in an outerplanar graph. Then color this graph by three colors. Since each triangle in the triangulation must have vertices of all three colors, putting a guard at each vertex in the smallest color class produces a covering by $\lfloor n / 3\rfloor$ guards.

\footnotetext{
* The author thanks The Fields Institute for Research in Mathematical Sciences and the Natural Sciences and Engineering Research Council of Canada for financial support.
} 


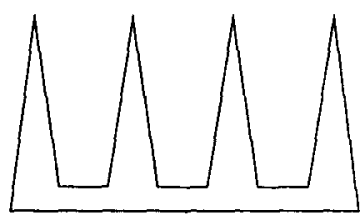

Fig. 1. An art gallery requiring four guards.

Hutchinson [4] and Griggs generalized the basic art gallery problem by allowing interior walls. Our notation follows [5]. An art gallery (with interior walls) is a simple polygon on $n$ vertices with $m$ pairs of vertices joined by nonintersecting interior diagonals - the interior walls. In each interior wall there is an arbitrarily placed, arbitrarily small opening (doorway). Figure 2 shows an example of an art gallery on $n=15$ vertices and $m=8$ interior walls that requires nine guards. Kündgen [5] found the number of guards that suffice to cover any such art gallery on $n$ vertices and with $m$ interior walls.

Theorem 1 [5]. The minimum number of guards that suffice to cover all art galleries with $n$ vertices and $m$ interior walls is

$$
g(n, m)= \begin{cases}\lfloor(2 n-3) / 3\rfloor & \text { for } m \geq\left\lfloor\frac{2}{3} n\right\rfloor-2, \\ \lfloor(2 m+n) / 3\rfloor & \text { for } m<\left\lfloor\frac{2}{5} n\right\rfloor, \\ \lfloor(2 n+m-2) / 4\rfloor & \text { otherwise. }\end{cases}
$$

\section{Displacing Checkpoints and Guards}

The arguments in the first two cases of Theorem 1 are similar to those in Fisk's proof, and they lead to linear-time guard placement algorithms. On the other hand, the proof of the upper bound in the third case given in [5] is inductive, and it is not known if it can be turned into a linear-time algorithm.

We present a "Fisk-type" proof of the upper bound $g(n, m) \leq\lfloor(2 n+m-2) / 4\rfloor$. This bound is valid for all values of $m, n$, however, it is optimal only in the range specified by Theorem 1 . The bound is a straightforward consequence (place guards to the points of the smallest color class) of the following lemma:

Lemma 2. Let $\mathcal{P}$ be an art gallery with $n$ vertices and $m$ interior walls, and let $\mathcal{P}_{\Delta}$ be an outerplanar triangulation of $\mathcal{P}$ respecting its interior walls. Then there exists a set

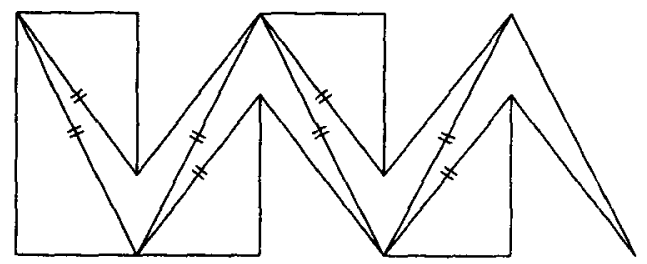

Fig. 2. An art gallery with interior walls requiring nine guards. 
$X$ of interior points of $\mathcal{P}$, and an incidence relation between $X$ and the triangular faces of $\mathcal{P}_{\Delta}$, satisfying the following:

1. Each point $x \in X$ guards all triangular faces of $\mathcal{P}_{\Delta}$ incident with $x$.

2. Each triangular face of $\mathcal{P}_{\Delta}$ is incident with exactly four points of $X$.

3. The set $X$ can be four-colored so that no two points incident with the same triangular face get the same color.

4. The cardinality of $X$ is $2 n+m-2$.

Proof. Throughout our proof we call the points of $X$ checkpoints. We call an edge of $\mathcal{P}_{\Delta}$ a wall if it is an outer or interior wall of $\mathcal{P}$, and we call it a nonwall if it is an edge introduced by the triangulation. A triangular face of $\mathcal{P}_{\Delta}$ is uniform if its edges are either all walls or all nonwalls. An angle at vertex $v$ of $\mathcal{P}$ determined by walls incident with $v$ and belonging to the interior of $\mathcal{P}$ is called a corner of $\mathcal{P}$. A corner at $v$ is divided if it contains a nonwall incident with vertex $v$ in $\mathcal{P}_{\Delta}$.

We introduce one checkpoint at every wall of $\mathcal{P}_{\Delta}$, one checkpoint in every divided corner, and one checkpoint in every uniform triangle of $\mathcal{P}_{\Delta}$. A "wall" checkpoint is incident with one or two triangles adjacent to the wall. A "corner" checkpoint is incident with all triangles spanning the corner. A "triangle" checkpoint is incident with its triangle. (Note that our incidence is an abstract relation, a checkpoint does not have to be an element of each triangle incident with it.) To fulfill the requirement that checkpoints belong to the interior of $\mathcal{P}_{\Delta}$, we place them as follows: For an interior wall, the checkpoint is placed in the middle of the doorway. For an outer wall, the checkpoint is placed at a close distance perpendicularly from the middle of the wall. For a divided corner, the checkpoint is put on the line of symmetry of the corner close to its vertex. See an example in Fig. 3.

It is now routine work to verify the validity of properties (1) and (2). Let $C(T) \subset$ $X$ denote the set of checkpoints incident with triangle $T$ (so $|C(T)|=4$ ). To prove (3), notice that the topological dual of $\mathcal{P}_{\Delta}$ is a tree, and hence the triangular faces of $\mathcal{P}_{\Delta}$ can be ordered $T_{1}, T_{2}, \ldots, T_{n-2}$ so that $T_{i}$ is adjacent to exactly one $T_{k_{i}}, k_{i}<i$

for $i=2, \ldots, n-2$. Since all checkpoints of $C\left(T_{i}\right) \cap \bigcup_{j=1}^{i-1} C\left(T_{j}\right)$ are incident with $T_{k_{i}}$, we can four-color $X$ by the greedy algorithm in order extending $C\left(T_{1}\right), C\left(T_{2}\right) \backslash$ $C\left(T_{1}\right), \ldots, C\left(T_{n-2}\right) \backslash \bigcup_{j=1}^{n-3} C\left(T_{j}\right)$.

Surprisingly, the last property (4) is the most difficult one. Clearly there are $m+n$ "wall" checkpoints. Next we show the following claim:

Claim 1. Each room of our gallery (i.e., interior face of $\mathcal{P}$ ) contains $t+2$ nondivided corners, where $t$ is the number of uniform triangles in this room.

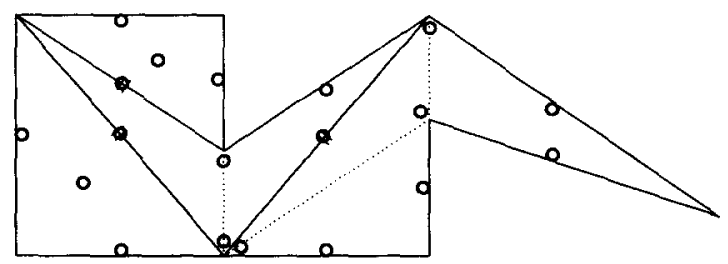

Fig. 3. Placement of 19 checkpoints for a gallery with $n=9, m=3$. 
The claim holds if the room is just a triangle. Otherwise, the topological dual of the room in $\mathcal{P}_{\Delta}$ is a tree $\mathcal{T}$ with maximal degree 3 . In this case every vertex of degree 1 in $\mathcal{T}$ corresponds to one nondivided corner, and every degree- 3 vertex of $\mathcal{T}$ corresponds to a uniform triangle. Hence the claim follows from the well-known equality $\sum_{v \in V(\mathcal{T})} d(v)=$ $2|V(\mathcal{T})|-2$

Now, our gallery has $m+1$ rooms that have $r_{j}$ vertices and $t_{j}$ uniform triangles, $j=1, \ldots, m+1$. An easy counting gives $\sum_{j=1}^{m+1} r_{j}=n+2 m$. By the above claim, the total number of divided corners in the gallery is

$$
\sum_{j=1}^{m+1}\left(r_{j}-t_{j}-2\right)=\sum_{j=1}^{m+1} r_{j}-\sum_{j=1}^{m+1^{j}} t_{j}-2(m+1)=n-2-\sum_{j=1}^{m+1} t_{j}
$$

Since there are $\sum_{j=1}^{m+1} t_{j}$ checkpoints placed for uniform triangles, the total number of checkpoints displaced in our construction is $m+n+n-2$.

If we have the triangulation $\mathcal{P}_{\Delta}$ at hand, the proof of Lemma 2 gives a straightforward algorithm to find the smallest color class $X_{0}$ of a four-coloring of $X$ in linear time. (However, notice that this algorithm just meets the general upper bound, it need not produce optimal guard placements in every particular case.) The problem to triangulate a given polygon can be, at least theoretically, solved also in linear time by the result of Chazelle [1]. For our purpose we need to apply the triangulation algorithm for each room separately, but that still leads to a linear overall computing time.

A little question remains about how to find the exact positions of the selected checkpoints $X_{0}$. For a wall or triangle checkpoint, we can easily compute the coordinates in constant time. Suppose that $x$ is a corner checkpoint at a corner with vertex $v$, and that $T_{1}, \ldots, T_{c}$ are the triangles incident with $x$. Our goal is to place $x$ as close to $v$ so that none of the straight lines supporting the edges of $T_{i}, i=1, \ldots, c$, not containing the vertex $v$ separates $x$ from $v$. For that we need time linear in $c$, but since every triangle of $\mathcal{P}_{\Delta}$ is counted only once, we can finish computation in linear time for the whole gallery.

\section{References}

1. B. Chazelle, Triangulating a simple polygon in linear time, Discrete Comput. Geom. 6 (1991), 485-524.

2. V. Chvátal, A combinatorial theorem in plane geometry, J. Combin. Theory Ser, B 18 (1975), 39-41.

3. S. Fisk, A short proof of Chvátal's watchman theorem, J. Combin. Theory Ser. B 24 (1978), 374.

4. J. Hutchinson, Art galleries with walls, Problem \#10478, Amer. Math. Monthly 102 (1995), 746.

5. A. Kündgen, Art galleries with interior walls, Discrete Comput. Geom. 22 (1999) 248-258.

6. J. O'Rourke, Art Gallery Theorems and Algorithms, The International Series of Monographs on Computer Science, The Clarendon Press, Oxford University Press, New York, 1987. 